\title{
(2) OPEN ACCESS \\ Targeting human plasmacytoid dendritic cells through BDCA2 prevents skin inflammation and fibrosis in a novel xenotransplant mouse model of scleroderma
}

\author{
Rebecca L Ross @ (1, ${ }^{1,2}$ Clarissa Corinaldesi, ${ }^{1}$ Gemma Migneco, ${ }^{1}$ Ian M Carr, ${ }^{3}$ \\ Agne Antanaviciute, ${ }^{3}$ Christopher W Wasson, ${ }^{1,2}$ Antonio Carriero $(1), 1,4$ \\ Jörg H W Distler (ㄷ) , ${ }^{5}$ Steve Holmes, ${ }^{6}$ Yasser M El-Sherbiny (1) , ${ }^{7,8}$ Clive S McKimmie, \\ Francesco Del Galdo (10) 1,2
}

\begin{abstract}
Handling editor Josef $S$
Smolen

- Additional material is published online only. To view, please visit the journal online (http://dx.doi.org/10.1136/ annrheumdis-2020-218439).
\end{abstract}

For numbered affiliations see end of article.

\section{Correspondence to}

Dr Rebecca L Ross and Professor Francesco Del Galdo, Leeds Institute of Rheumatic and Musculoskeletal Medicine, Faculty of Medicine and Health, University of Leeds, Leeds, LS9 7TF, UK; r.l.ross@leeds.ac.uk, f.delgaldo@leeds.ac.uk

RLR and CC are joint first authors.

Work has been presented previously at American College of Rheumatology 2018 and World Scleroderma Foundation 2019.

Received 29 June 2020 Revised 7 January 2021 Accepted 9 January 2021 Published Online First 4 February 2021

\section{Check for updates}

(c) Author(s) (or their employer(s)) 2021. Re-use permitted under CC BY-NC. No commercial re-use. See rights and permissions. Published by BMJ.

To cite: Ross RL, Corinaldesi C, Migneco $\mathrm{G}$, et al. Ann Rheum Dis 2021:80:920-929.

\section{ABSTRACT}

Objectives Plasmacytoid dendritic cells ( $p D C$ ) have been implicated in the pathogenesis of autoimmune diseases, such as scleroderma (SSc). However, this has been derived from indirect evidence using ex vivo human samples or mouse pDC in vivo. We have developed human-specific pDC models to directly identify their role in inflammation and fibrosis, as well as attenuation of $\mathrm{pDC}$ function with BDCA2-targeting to determine its therapeutic application.

Methods RNAseq of human pDC with TLR9 agonist ODN2216 and humanised monoclonal BDCA2 antibody, CBS004. Organotypic skin rafts consisting of fibroblasts and keratinocytes were stimulated with supernatant from TLR9-stimulated pDC and with CBSO04. Human pDC were xenotransplanted into Nonobese diabetic/ severe combined immunodeficiency (NOD SCID) mice treated with Aldara (inflammatory model), or bleomycin (fibrotic model) with CBSO04 or human IgG control. Skin punch biopsies were used to assess gene and protein expression.

Results RNAseq shows TLR9-induced activation of human pDC goes beyond type I interferon (IFN) secretion, which is functionally inactivated by BDCA2 targeting. Consistent with these findings, we show that BDCA2-targeting of pDC can completely suppress in vitro skin IFN-induced response. Most importantly, xenotransplantation of human $\mathrm{PDC}$ significantly increased in vivo skin IFN-induced response to TLR agonist and strongly enhanced fibrotic and immune response to bleomycin compared with controls. In these contexts, BDCA2-targeting suppressed human pDCspecific pathological responses.

Conclusions Our data indicate that human pDC play a key role in inflammation and immune-driven skin fibrosis, which can be effectively blocked by BDCA2-targeting, providing direct evidence supporting the development of attenuation of pDC function as a therapeutic application for SSc.

\section{INTRODUCTION}

Plasmacytoid dendritic cells (pDC), specialised in the secretion of type I interferon (IFN), ${ }^{1-3}$ activate inflammatory responses through TLR-mediated sensing of nucleic acids released from pathogens during infection or following cell death in autoimmunity. ${ }^{4-8}$ Self-derived nucleic acids released from

\section{Key messages}

What is already known about this subject?

- Research to date on plasmacytoid dendritic cells (pDC) has focused only on indirect evidence using ex vivo human samples or mouse models, but suggests $\mathrm{pDC}$ involvement in interferon (IFN)-induced response, skin infiltration and fibrosis within immune-mediated inflammatory disease (IMID), such as scleroderma.

What does this study add?

- We devised a novel mouse model of human pDC through xenotransplantation of human pDC into immunocompromised mice, showing a significantly increased IFN-induced response to topical TLR agonist application and a strongly enhanced fibrotic and immune response to bleomycin, all of which were strongly suppressed by specific pDC BDCA2-targeting.

- We demonstrate directly that functional inactivation of human pDC through BDCA2targeting suppresses the entire TLR9-induced transcriptome, which includes type I IFN activation and a multitude of genes that could contribute to immune-driven tissue damage.

How might this impact on clinical practice or future developments?

- These data offer the first direct evidence supporting the development of BDCA2targeting as a therapeutic application for pDCmediated skin inflammation and fibrosis.

- The development of a human pDC mouse model will allow the expansion into other IMID to confirm pDC involvement.

damaged tissues, apoptotic/necrotic cells or bound to autoantibodies, can be recognised by TLR7/8/9 and have been shown to induce pDC activation and IFN secretion. ${ }^{9-15}$ The role of CXCL4 has been elucidated as an amplifier of TLR9-mediated pDC hyperactivation and IFN production by organising self-DNA into liquid crystalline immune complexes. $^{16}$

TLR-induced activation of pDC triggers stable cell differentiation into three subtypes, with 
PD-L1(CD274) ${ }^{+} \mathrm{CD}_{00}^{-}(\mathrm{P} 1)$ and PD-L1 ${ }^{+} \mathrm{CD} 80^{+}(\mathrm{P} 2)$ subpopulations specialised in type I IFN production both in healthy volunteers (HV) and patients with autoimmune conditions. ${ }^{17}$ pDC have been further implicated in the pathogenesis of autoimmune diseases, such as scleroderma (SSc), systemic lupus erythematosus (SLE) and psoriasis, through their ability to infiltrate the skin and secrete IFNs and proinflammatory chemokines. ${ }^{13}$ 18-22 Specifically, SSc is an immune-mediated inflammatory disease (IMID) characterised by vascular and tissue fibrosis, leading to diverse life-altering and life-threatening clinical manifestations. ${ }^{23}$ $\mathrm{pDC}$ have been observed in affected skin of patients with SSc, and purified peripheral SSc pDC have been shown to spontaneously produce higher levels of type I IFN compared with $\mathrm{HV}^{24-26}$ Indeed, an elevated IFN gene signature in affected organs and in the blood is a common feature of severe disease in SSc, ${ }^{25} 27$ which is present before the onset of clinical fibrosis. ${ }^{28}$ These observations collectively support the notion that $\mathrm{pDC}$ activation and type I IFN play an important role in SSc pathogenesis.

BDCA2 is a type II transmembrane glycoprotein that belongs to the C-type lectin superfamily receptor that can signal to inhibit pDC type I IFN secretion. ${ }^{29-31}$ BDCA2 signals through an associated transmembrane adaptor, the $\mathrm{F} \in \mathrm{R} \gamma$, which recruits the protein tyrosine kinase Syk, inducing protein tyrosine phosphorylation and calcium mobilisation, ${ }^{32}$ which reduces TLRinduced activation of pDC, inhibiting type I IFN secretion and other inflammatory mediators. ${ }^{30}$ 32-34 In SLE clinical trials, BDCA2-targeting antibodies induced a significant but partial decrease in IFN response within the blood, and reduced type I IFN-induced response and immune infiltrates in skin lesions. ${ }^{35} 36$ Recently, pDC's role in fibrosis was elucidated as elimination of mouse pDC reduced bleomycin-induced skin fibrosis, ${ }^{24}$ further highlighting the therapeutic potential of BDCA2-targeting for SSc. However, exploring the efficacy of BDCA2-targeting during fibrosis is difficult, as BDCA2 is only expressed in primates, highlighting the need for a human-specific pDC in vivo model. Furthermore, there are key differences between mouse and human $\mathrm{pDC}$; thus, functions determined in mouse models may not be fully transferable to human pDC. ${ }^{13}{ }^{37-39}$

We developed human-specific models to uncover the role of pDC biology in inflammation and fibrosis, as well as attenuation of $\mathrm{pDC}$ function with BDCA2-targeting to determine its therapeutic application for SSc.

\section{RESULTS}

\section{TLR9-induced activation of human pDC goes beyond type I IFN secretion and is hindered by BDCA2-targeting}

Using RNAseq, we set out to discover the transcriptome of human pDC when stimulated with TLR9 agonist, A-class oligodeoxyribonucleotides containing CpG motifs (ODN2216/ ODN), to understand the pathways that could contribute to the pathogenesis of chronic inflammation and immune-driven tissue damage seen in IMID. We performed RNAseq analysis of human pDC purified from peripheral blood mononuclear cells (PBMC) from four HV (online supplemental figure S1), as previously described. ${ }^{34}$ Transcriptome analysis revealed 328 differentially expressed genes (DEGs $\geq$ or $\leq 2$ fold change, $\mathrm{FDR} \leq 0.05$ ) between unstimulated (control/CTR) and ODN-stimulated pDC $(\mathrm{ODN})$, with donor heterogeneity observed with ODN stimulation, suggesting $\mathrm{pDC}$ response donor variability (figure $1 \mathrm{~A}$ and online supplemental table 1). Pathway analysis identified genes involved in immune response against viruses and other organisms as key enriched biological processes (figure 1B). These innate immune processes match to those previously identified in characterised inflammatory SSc skin subsets, ${ }^{40}$ suggesting involvement of pDC activation in this specific subset. Consistent with this notion, we observed upregulation of many type I IFNdependent pathways and IFN-related genes, such as IFN-A2, IFN-A21, IFN-B1 and CCL5, a common feature seen in SSc. ${ }^{40-42}$ Pathway analysis also showed JAK/STAT, nuclear factor kappa B subunit 1 (NF- $\mathrm{KB}$ ) and angiogenesis pathways to be major biological processes upregulated by ODN stimulation (figure 1B), which have been shown to be dysregulated in SSc ${ }^{13} 4041$ but not shown in $\mathrm{pDC}$ before. These data suggest that TLR stimulation of pDC can induce a multitude of genes beyond IFN, which could contribute to the pathogenesis of inflammation in SSc and other IMID. Interestingly, a recent publication showed that the majority of SSc skin samples with higher fibroblast scores had significantly increased macrophage and/or dendritic cell scores, suggesting a link between the two cell types that are important for inducing the fibroinflammatory signature. ${ }^{41}$

A monoclonal BDCA2 antibody (clone AC144) has previously been shown to suppress human pDC TLR-induced IFN type I secretion by interfering with the Fc€R $\gamma$-Syk signalling. ${ }^{19} 30$ To aid our understanding of human $\mathrm{pDC}$ in IMID, we generated mouse monoclonal antibodies $(\mathrm{mAb})$ against human BDCA2 and fully humanised the lead mAb, CBS004, which had a greater affinity for BDCA2 compared with AC144 control (online supplemental figure S2A). Ex vivo direct competition assays showed that CBS004 and AC144 bind alternative pDC epitopes, as indicated by double staining of the $\mathrm{pDC}$ population gated within PBMC $\left(\mathrm{LIN}^{-} \mathrm{HLA}^{+} \mathrm{CD} 123^{+} \mathrm{CD} 304^{+}\right.$) (online supplemental figure S2B,C).

BDCA2-targeting using CBS004 reduced the expression of $60 \%$ of ODN-inducible DEGs $\geq 1.5$-fold (figure $1 \mathrm{C}$ and online supplemental table 2). It has been recently shown that TLR-induced pDC triggers stable cell differentiation into three stable subtypes, with PD-L1(CD274) ${ }^{+} \mathrm{CD} 80^{-}(\mathrm{P} 1)$ and PD-L1(CD274) ${ }^{+} \mathrm{CD} 80^{+}$ (P2) subpopulations specialised in type I IFN production both in $\mathrm{HV}$ and patients with autoimmune conditions. ${ }^{17}$ Consistent with these observations, ODN induced CD274 expression, which was also suppressed by BDCA2-targeting (figure 1C). ${ }^{17}$ To validate the RNAseq findings at protein level, we measured the PD-L1 and CD80 positive subpopulations by fluorescenceactivated cell sorting (FACS) (figure 1D,E). Similarly to what has been observed following viral stimulation, ${ }^{17}$ ODN induced $62 \%$ of pDC differentiation into P1 and P2, with no differences observed between healthy and SSc samples (figure 1D,E). In this context, BDCA2-targeting caused an increase of the P3 subpopulation, which has been previously shown to produce less IFN. ${ }^{17}$ These data were further validated by performing FACS analysis of IFN-positive pDC gated within human PBMC (online supplemental figure S2B). Functionally, ODN led to a dramatic induction of IFN positive $\mathrm{pDC}$, which was reduced by $76 \%$ with BDCA2-targeting (figure 1F,G).

Beyond type I IFN signature genes, ODN-induced inflammatory interleukin (IL)-6 expression was also modulated by BDCA2targeting. Interestingly, IL-6 and IFN secretion can synergistically activate B cells. ${ }^{17}{ }^{43}$ Among other genes of interest, serglycin (SRGN) also showed a TLR-induced and BDCA2-dependent pattern. SRGN has been shown to be secreted into the extracellular matrix and linked to promoting lymphoid cells adhesion and activation, ${ }^{44} 45$ storage of chemokines and cytokines, as well as being able to induce epithelial-mesenchymal transition. ${ }^{46} 47$ These data demonstrate that TLR stimulation of human $\mathrm{pDC}$ goes beyond IFN secretion induction and predicts a greater biological relevance of $\mathrm{pDC}$ activation in IMID. Our analyses show that TLR-induced pDC activation can be drastically suppressed by BDCA2-targeting. 
A

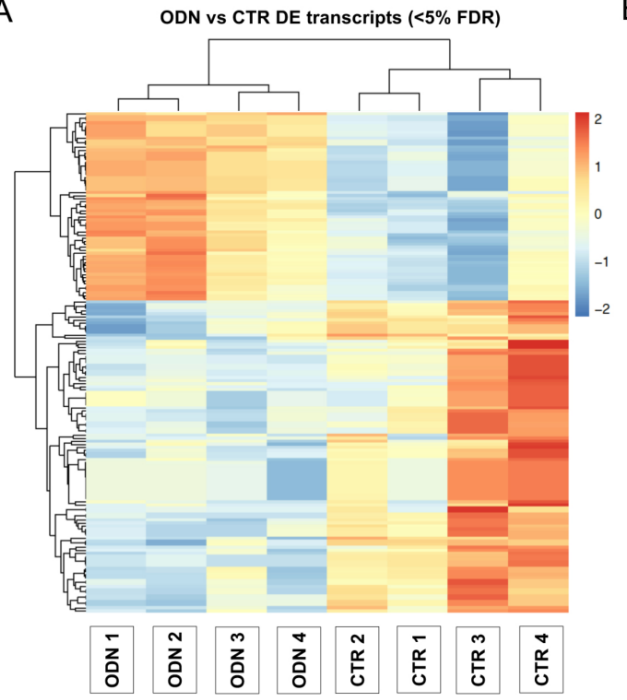

B

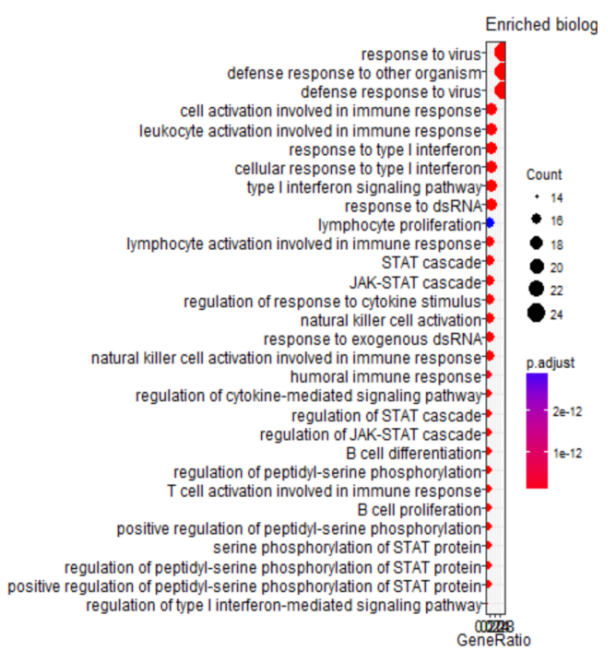

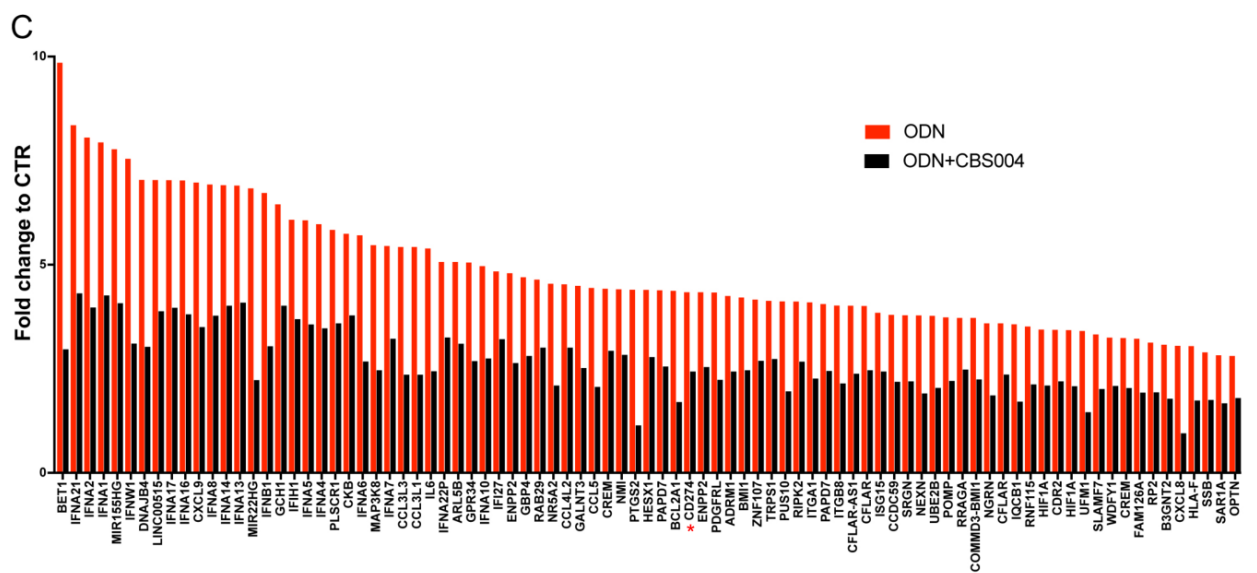
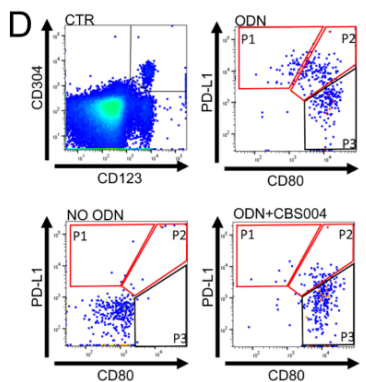

$\mathrm{E}$
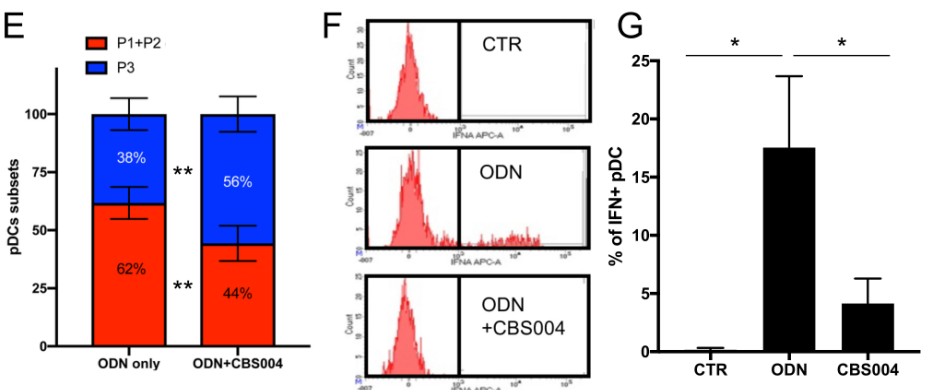

Figure 1 TLR9-induced activation of human PDC goes beyond type I IFN secretion and is hindered by BDCA2-targeting in vitro. Transcriptome analysis of human pDC cultured in media alone (CTR), with $1 \mu \mathrm{M}$ ODN2216 (ODN) or with ODN and CBS004 (10 $\mu \mathrm{g} / \mathrm{mL}$ ) (added 15 min prior to stimulation) for 16 hours $(n=4)$. (A) Heatmap of reduced, centred normalised read counts for DE transcripts among CTR and ODN populations, $<5 \%$ FDR, calculated using Benjamini-Hochberg multiple testing correction. DE transcripts $\geq 0$ r $\leq 2$-fold (FDR $<0.05)$ shown in online supplemental table 1. (B) Kyoto Encyclopedia of Genes and Genomes (KEGG) pathway analysis showing top biological processes enriched in the set of DE transcripts between CTR and ODN pDC, and their associated $p$ values. (C) Average fold change of DEGs for ODN and ODN+CBS004 relative to CTR $(n=4)$. For repeat transcripts, the highest fold change was used for comparison. Red bars represent the 87 genes that were increased $\geq 2$-fold (FDR $<0.05$ ) between CTR and ODN that are dependent on BDCA2 treatment (reduced $\geq 1.5$-fold by CBS004) (full transcript data, online supplemental table 2). $(D, E)$ Validation of CD274 (PD-L1) TLR-induced expression. (D) Example of subtyping FACS analysis of pDC (CD123 $3^{+}$DD204 $\left.{ }^{+}\right)$in the three culture conditions (CTR, ODN and ODN+CBS004). Gating illustrates P1, P2 and P3 subtypes, with the former two previously shown to be IFN-secreting cells. ${ }^{17}$ PBMC were cultured for 16 hours and pDC sorted as Lin $\mathrm{HLA}^{-} \mathrm{DR}{ }^{+} \mathrm{CD} 45^{+} \mathrm{CD} 123^{+} \mathrm{CD} 304^{+}$(online supplemental figure S2B) and gated for PD-L1 and $\mathrm{CD} 80$ expression to determine $\mathrm{P1}$, P2 and P3 sub-types. P1, PD-L1 ${ }^{+} \mathrm{CD} 80^{-} ; \mathrm{P2}, \mathrm{PD}-\mathrm{L1}^{+} \mathrm{CD} 80^{+} ; \mathrm{P} 3, \mathrm{PD}-\mathrm{L} 1{ }^{-} \mathrm{CD} 80^{+} .{ }^{17}$ (E) Quantification of sub-types based on FACS analysis of PBMC samples (healthy and SSC $n=3,4$ ) between ODN and ODN+CBS004 culture conditions. (F,G) Validation of IFN-related gene expression. (F) Representative histogram of intracellular IFN alpha staining of pDC gated within PBMC when cultured in RPMI alone, with ODN (1 $\mu \mathrm{M})$ and with CBS004 $(10 \mu \mathrm{g} / \mathrm{mL})$. (G) Percentage of IFN-positive $\mathrm{pDC}$ from FACS analysis from $\mathrm{F}(\mathrm{n}=6)$. ( $(\mathrm{E}, \mathrm{F})$ Error bars represent mean $\pm S E M$, and statistical significance was evaluated using paired two-tailed t-test. ${ }^{*} P<0.05$, ${ }^{*} P<0.01$. CTR, control; $D E$, differentially expressed; $D E G$, differentially expressed gene; FACS, fluorescence-activated cell sorting; FDR, false discovery rate; IFN, interferon; PBMC, peripheral blood mononuclear cell; pDC, plasmacytoid dendritic cell; SEM, standard error of mean; SSc, scleroderma. 
Human $\mathrm{pDC}$ induced IFN response in organotypic skin rafts (OSR), which is inhibited by BDCA2-targeting

Growing evidence shows pDC skin infiltration and induced IFN signature within the skin of patients with IMID, such as SSc. ${ }^{24-28}$ Furthermore, PBMC-conditioned media are known to activate a proinflammatory response in fibroblasts. ${ }^{48}$ To determine whether TLR9-stimulated pDC could also induce this response, and whether treatment with CBS004 may have a functional effect on target tissue cell activation, we set out to measure IFN-induced genes in an OSR model of keratinocytes and fibroblasts co-culture following exposure to $\mathrm{pDC}$ supernatants (figure $2 \mathrm{~A}$ ).

First, we performed IFN secretion ELISA assays on pDC purified from PBMC (online supplemental figure S1) to establish the concentration of IFN within ODN-stimulated pDC supernatants. ODN stimulation was for 16 hours to allow IFN secretion into the supernatant to accumulate, since pDC-IFN maximum production has previously been shown between 12 and 18 hours. ${ }^{495}$ TLR9 induced a striking increase in IFN- $\alpha$ secretion. The addition of CBS004 15 min prior to ODN stimulation suppressed this by $90 \%$, whereas human $\operatorname{IgG}(\mathrm{HIgG}$ ) had no significant effect (figure 2B). To ensure functional inhibition driven by BDCA2-targeting of human $\mathrm{pDC}$ was maintained in HV and SSc PBMC, we conducted the same experiment ex vivo. ODN stimulation of PBMC induced a similar but substantial increase in IFN secretion in HV and SSc samples, which again was suppressed by $>98 \%$ in all samples by BDCA2-targeting (figure 2C). To determine the dose-response of CBS004 and to determine the concentration needed for maximal inhibition of IFN secretion, similar experiments were carried out on ODN-treated HV PBMC, identifying IC50 $0.06 \mathrm{nM}$ and IC90 $0.86 \mathrm{nM}$ values, with HIgG not effecting IFN production (figure 2D). The inhibitory activity of CBS004 was 17 -fold higher than previously tested $\mathrm{mAb}$ AC144, which supports the in vitro data showing enhanced BDCA2 affinity and binding (online supplemental figures S3A and S2A). Importantly, BDCA2-targeting did not significantly reduce pDC viability as determined by 7AAD assay gated within HV PBMC (online supplemental figures S2B, S3B).

OSR were treated with supernatants from $\mathrm{pDC}$ cultured in cell media alone (CTR), ODN or ODN+CBS004 (figure $2 \mathrm{~A}, \mathrm{~B})$. The volume of supernatant was calculated to produce a final concentration of $6000 \mathrm{pg} / \mathrm{mL}$ of IFN in the ODN experiment, as determined via ELISA (figure 2B). Real-time quantitative reverse transcription PCR (qRT-PCR) of 78 key interferon signalling genes (ISGs) was performed on RNA collected from the keratinocyte and fibroblast collagen matrix. ODN supernatants resulted in an increase of 1.8fold to 32-fold in 35 ISGs relative to CTR (figure 2E). Despite the limited number of models tested $(n=3)$, eight genes showed a statistically significant upregulation, including ISG15, IFITM1, BST2, IFI6, IFIH1, NMI, HLA-B and IFITM3 (induction of 3-fold to 19 -fold relative to control and $\mathrm{p}<0.05$ ). These data support the use of OSR to explore the effect of TLR-activated $\mathrm{pDC}$ in a preclinical human model. Importantly, ODN+CBS004 supernatants resulted in suppressed upregulation in all of those genes, ranging from 1.8fold to 11-fold compared with gene expression induced by ODN (figure 2F; ANOVA, $\mathrm{p}<0.0001$ ). This resulted in a transcription profile similar to CTR (figure 2G). Together these results suggest that BDCA2-targeting of $\mathrm{pDC}$ can suppress the IFN signature within skin cells.

\section{Xenotransplant of human pDC in NOD SCID mice increased skin IFN response to TLR stimulation in a BDCA2-dependent manner}

To advance our understanding of the role of circulating human pDC in IFN-induced response within the skin, we developed a novel in vivo model. We implemented a xenotransplant transfer of purified normal human primary pDC into nonobese diabetic/severe combined immunodeficiency (NOD SCID) mice (XenoSCID) via intravenous (iv) injection followed by topical application of imiquimod-containing cream (Aldara, TLR7 agonist), with or without anti-BDCA2 (CBS004) or human IgG (HIgG) (online supplemental figure S4A). Topical imiquimod contains a TLR7 agonist that when applied to resting skin induces expression of type I IFN, primarily in macrophages. ${ }^{51}$ Repeated application of topical imiquimod results in induction of ISG in an IFNAR1-dependent manner, the recruitment of leukocytes, skin thickening and the development of an inflammatory lesion. Xenotransplantation of human pDC into this model would allow us to determine $\mathrm{pDC}$ role in ISG response to imiquimod and whether it is sensitive to BDCA2-targeting. In vitro imiquimod-stimulation of healthy PBMC induced IFN secretion, which was BDCA2-dependent (online supplemental figure $\mathrm{S4B}$ ). The purity of $\mathrm{pDC}$ isolated from healthy $\mathrm{PBMC}$ for in vivo experiments and functional responses to TLR-9 were assessed (online supplemental figures S1, S4C). FACS analysis of $\mathrm{CD} 45^{+} \mathrm{CD} 123^{+} \mathrm{CD} 304^{+}$cells indicated pDC skin infiltration within the Aldara+pDC condition $(0.3 \%$ of total cells, figure $3 \mathrm{~A}$ ), which resulted in a functional increase in mouse skin ISG expression, as mice receiving human $\mathrm{pDC}$ induced a 3.2-fold increase in composite ISG score (including Ifit1, Isg15, Mx1, Cxcl10 and Viperin), compared to CTR (ANOVA P<0.05) (figure 3B). Importantly, this is greater than Aldara treatment alone in absence of $\mathrm{pDC}$, where a 1.7-fold increase in composite ISG score compared to CTR was observed (figure 3B). In agreement, IHC staining showed only a slight increase in MX1 protein expression levels between CTR and Aldara (online supplemental figure S4D). Interestingly, epidermal thickening was observed (online supplemental figure S4D), which has previously been shown be an Aldara-induced response independent of type I IFN and TLR7. ${ }^{38}$

In this context, we could assess the in vivo efficacy of BDCA2targeting. CBS004 and $\mathrm{HIgG}$ control $\mathrm{mAb}$ were injected into XenoSCID 12 hours before pDC intravenous injection (online supplemental figure S4A). Aldara-induced pDC skin infiltration, as detected by human $\mathrm{CD} 45^{+} \mathrm{CD} 123^{+} \mathrm{CD} 304^{+}$cells in the mouse treated skin, was not hindered by HIgG $(0.3 \%$ of total cells) but reduced 3-fold by BDCA2-targeting (figure 3C). Most importantly, BDCA2-targeting suppressed 44\% of the ISG expression observed in Aldara+pDC, which was significantly reduced compared with HIgG administration (figure 3D).

Consistent with these findings, immunohistochemistry (IHC) analysis showed a strong induction of MX1 and pSTAT1 (Tyr701) with pDC transplantation compared with NOD SCID mice, which was dramatically reduced by BDCA2-targeting and unaffected by HIgG (figure 3E,F).

\section{Xenotransplant of human pDC in NOD SCID mice increased the skin profibrotic response to bleomycin treatment in a BDCA2-dependent manner}

Ah Kioon et al have shown that depletion of mouse pDC can ameliorate bleomycin-induced skin fibrosis in a mouse model of SSc. ${ }^{24}$ While supporting the role of pDC in fibrosis in mice, the model could not directly demonstrate the role of human pDC in this setting. Thus, we developed our XenoSCID model with bleomycin-induced skin fibrosis. We supplemented every other day subcutaneous injection of bleomycin with weekly tail vein injection of human pDC for 3 weeks (online supplemental figure $\mathrm{S} 5 \mathrm{~A}$ ). As anticipated in an immunocompromised mouse, bleomycin alone induced a blunted fibrotic response at 3 weeks, 

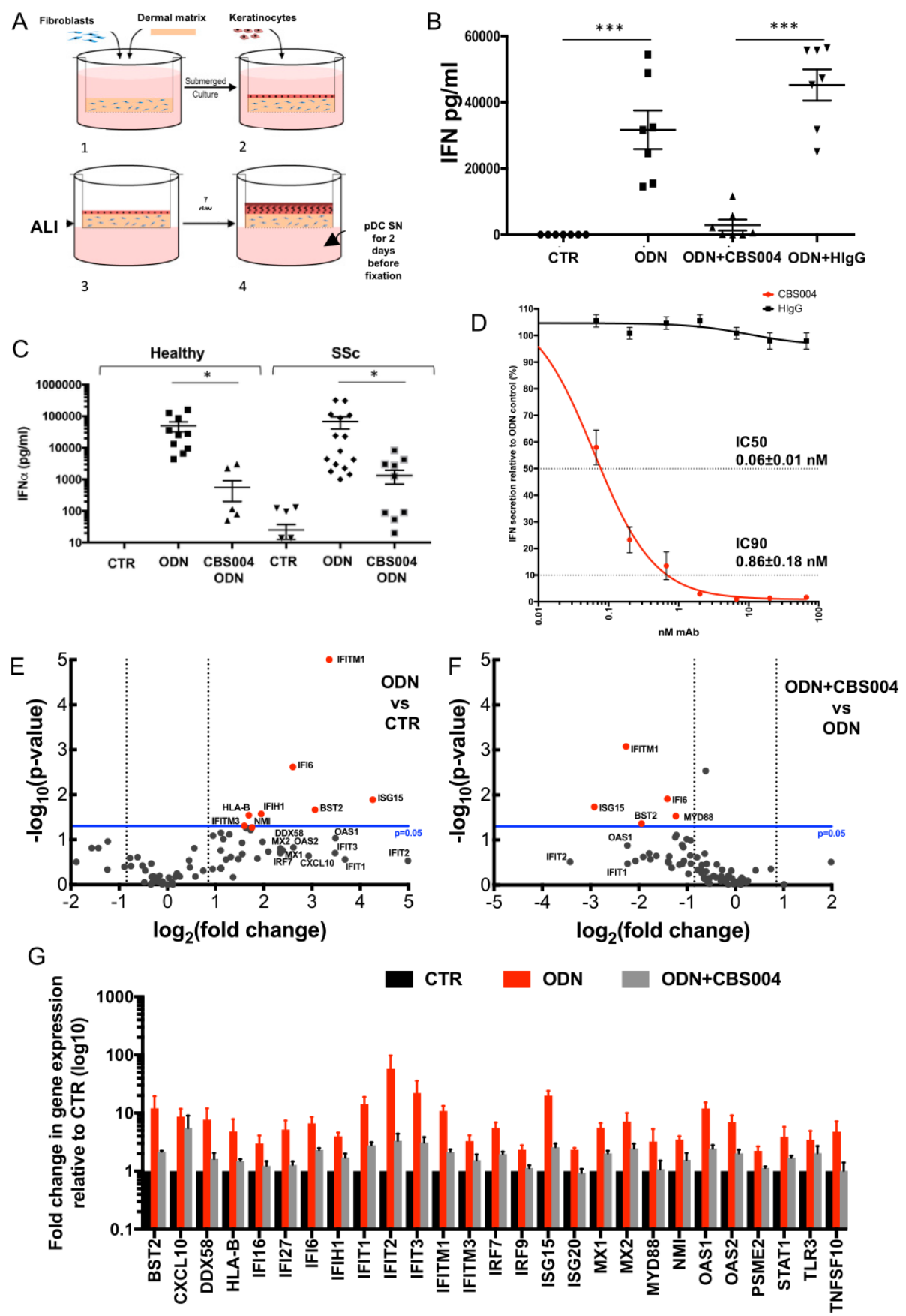

Figure 2 Human pDC induced IFN response in OSR, which is suppressed by BDCA2-targeting. (A) Systematic outline of OSR protocol; fibroblasts are embedded into a collagen matrix and keratinocytes seeded above until confluence. OSR is brought to ALI to sustain epithelium differentiation. After 5 days, ALI media spiked with 6000 pg/mL of IFN (generated by TLR-stimulated pDC, ODN (B)) for 48 hours. CTR contains equivalent supernatant from untreated pDC (undetectable IFN) and from pDC treated with ODN+CBS004 (10 $\mu \mathrm{g} / \mathrm{mL})$. (B) Quantification of IFN secretion from purified HV pDC $(n=7)$ after 16 hours of culturing in cell media alone (CTR), with ODN $(1 \mu \mathrm{M})$ and with CBS004 or human lgG1 (10 $\mu \mathrm{g} / \mathrm{mL})(\mathrm{added} 15 \mathrm{~min}$ prior to stimulation) measured by ELISA to determine volume to add to ALI. (C) CBS004 suppresses TLR-induced IFN secretion in HV and SSC PBMC. PBMC from donors were cultured in media alone (CTR), with $1 \mu \mathrm{M}$ ODN, or with ODN and CBS004 [10 $\mu \mathrm{g} / \mathrm{mL}]$ for 16 hours ( $\mathrm{n}=15)$. IFN was quantified in the supernatants by ELISA. (B,C) Error bars represent mean \pm SEM and statistical significance was evaluated using unpaired two-tailed t-test. (D) Percentage of IFN alpha secretion, measured by ELISA, from PBMC from four donors stimulated with ODN in the presence of CBS004 or HIgG (0-66 $\mathrm{nM}$ ) relative to ODN-stimulated pDC with no antibody (100\%). Dotted lines highlight IC50s and IC90 with mean values \pm SEM. (E,F) RNAs harvested from $3 \mathrm{~mm}$ biopsies from OSR and subjected to type I IFN inducible genes superarray. Volcano plots illustrate the fold change of 79 IFN type I-related genes (black dots) between CTR and ODN (E) and between ODN and ODN+CBS004 (F) (pDC supernatant from three different donors for each condition). Grey lines represent the 1.8-fold change cut-off. The blue line represents the cut-off for statistical significance of $p=0.05$ calculated using Student's t-test (two-tailed distribution and equal variances between the two samples) on the triplicate $2-\Delta \mathrm{Ct}$ values for each gene in each treatment group compared with the CTR group. (F) bar chart illustrates the IFN type I-related genes that were $>1.8$ fold increased in ODN relative CTR and the effect of CBS004. results are represented as means \pm SEM. ${ }^{*} \mathrm{P}<0.05,{ }^{* *} \mathrm{P}<0.001$. ALI, air-liquid interface; CTR, control; HIgG, human IgG; HV, healthy volunteer; IFN, interferon; OSR, organotypic skin raft; PBMC, peripheral blood mononuclear cell; pDC, plasmacytoid dendritic cell; SEM, standard error of mean; SSc, scleroderma. 


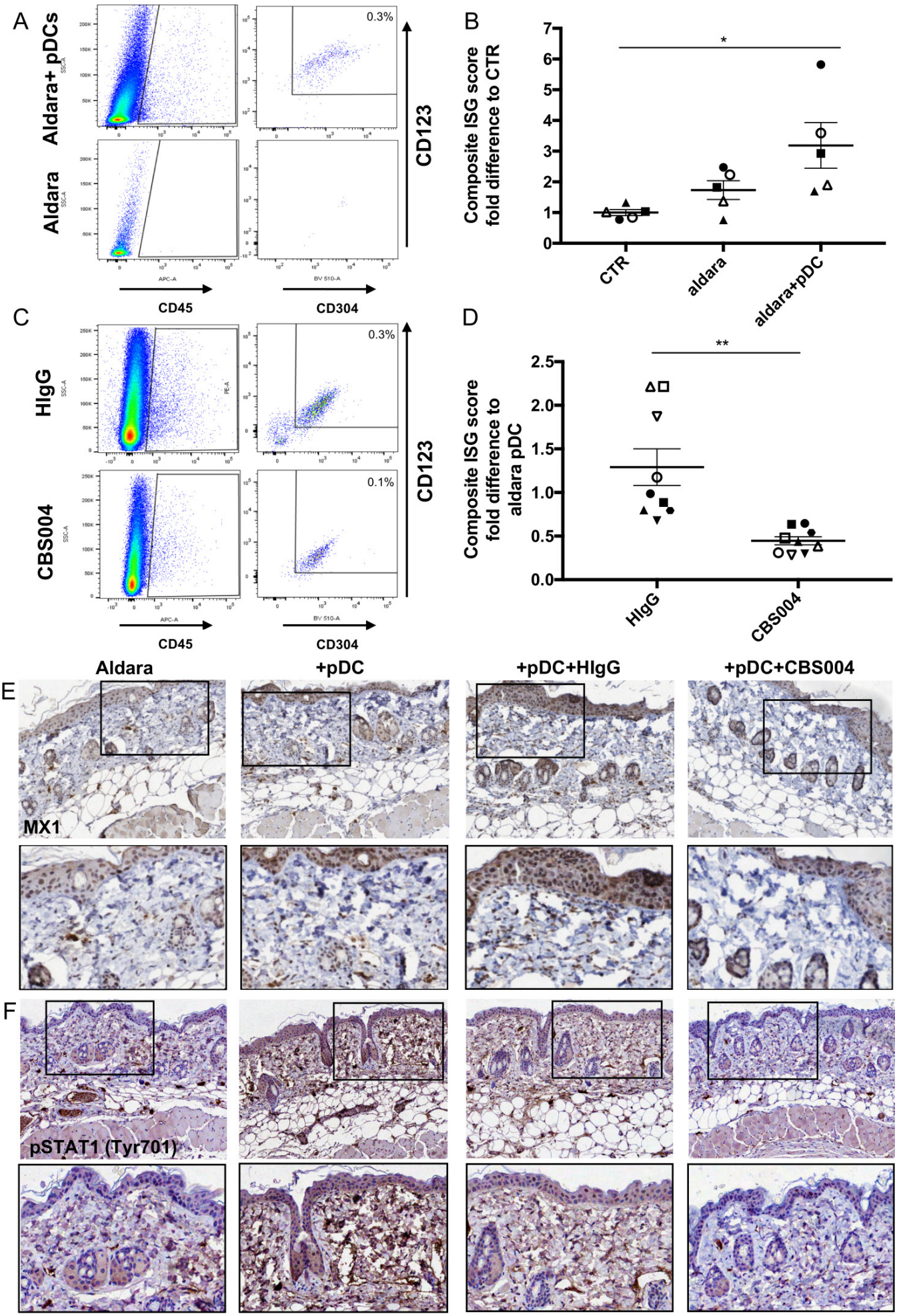

Figure 3 XenoSCID with human pDC increased skin IFN response to TLR stimulation in a BDCA2-dependent manner. Intravenous tail injection of $2.5 \times 10^{5}$ human purified pDC and intraperitoneal injection of CBS004 mAb (5 mg/kg) or CTR human lgG to NOD SCID mice treated with topical Aldara cream application (online supplemental figure S4A, systematic diagram and timeline), with five different treatment conditions consisting of CTR ( $\mathrm{n}=5$ ), Aldara $(n=5)$, Aldara+pDC $(n=5)$, Aldara+pDC+CBS004 $(n=9)$ and Aldara+pDC+HIgG $(n=9)$. Treated skin was harvested using a 3 mm punch biopsy and processed for FACS analysis of human pDC (CD45 $\left.{ }^{+} \mathrm{CD} 123^{+} \mathrm{CD} 304^{+}\right)$(representative analyses $\mathrm{A}$ and $\left.\mathrm{C}\right)$, qRT-PCR analysis for type I IFN inducible genes (B,D), and IHC staining for MX1 and pSTAT1 (Tyr701) (E,F). (B) Composite ISG score within Aldara and Aldara+pDC conditions relative to CTR. Score shows average fold difference between relative expression of $M x 1$, Isg15, CxCl10, Ifit1, Isg15 and Viperin between the test condition and CTR. Different symbols represent the different mice litters/human pDC donors. Statistical significance was evaluated using analysis of variance test. (D) Illustration of the composite ISG scores for $+\mathrm{CBSO04}$ and $+\mathrm{HlgG}$ conditions relative to that of Aldara+pDC. Statistical significance was evaluated using unpaired two-tailed t-test, ${ }^{*} \mathrm{P}<0.05$, ${ }^{*} \mathrm{P}<0.01$. CTR, control; HIgG, human IgG; IHC, immunohistochemistry; ISG, interferon signalling gene; NOD $S C I D$, nonobese diabetic severe combined immunodeficiency; PDC, plasmacytoid dendritic cell; qRT-PCR, real-time quantitative reverse transcription PCR; NOD SCID. 

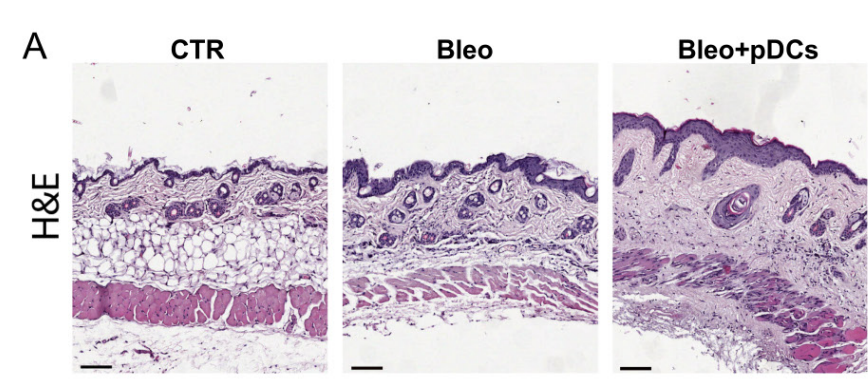
Bleo+pDCs
HIgG

\section{Bleo+pDCs}

CBS004

B
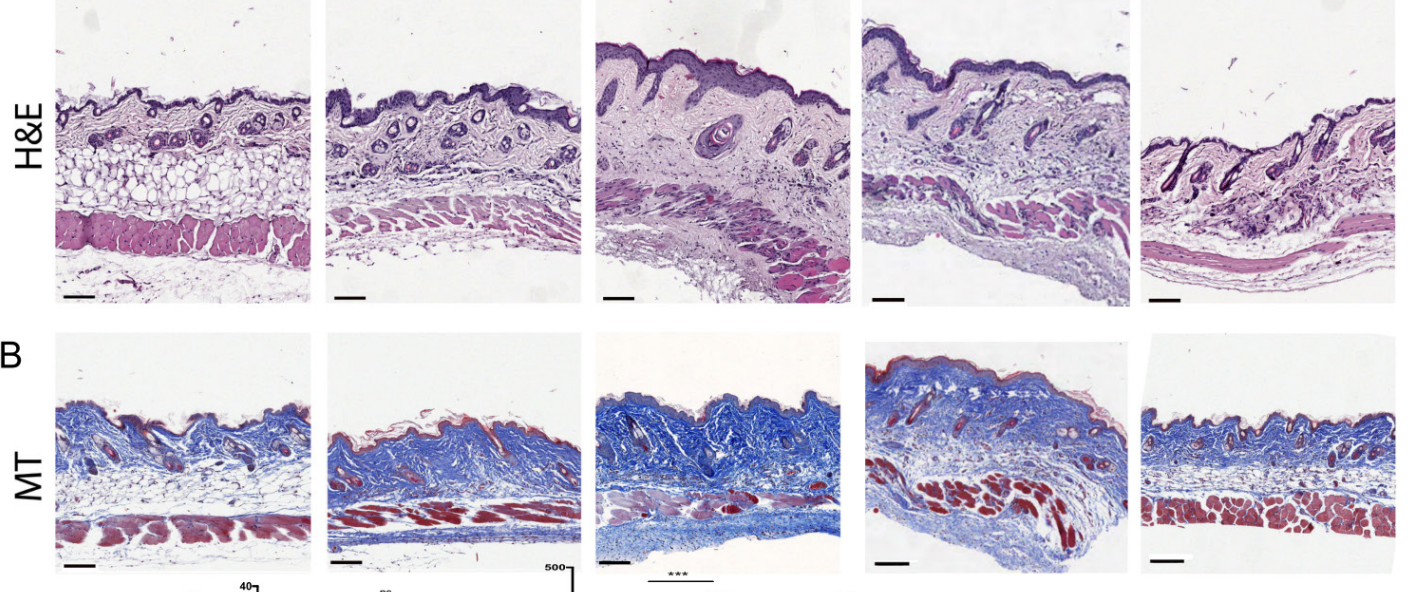

\section{C}
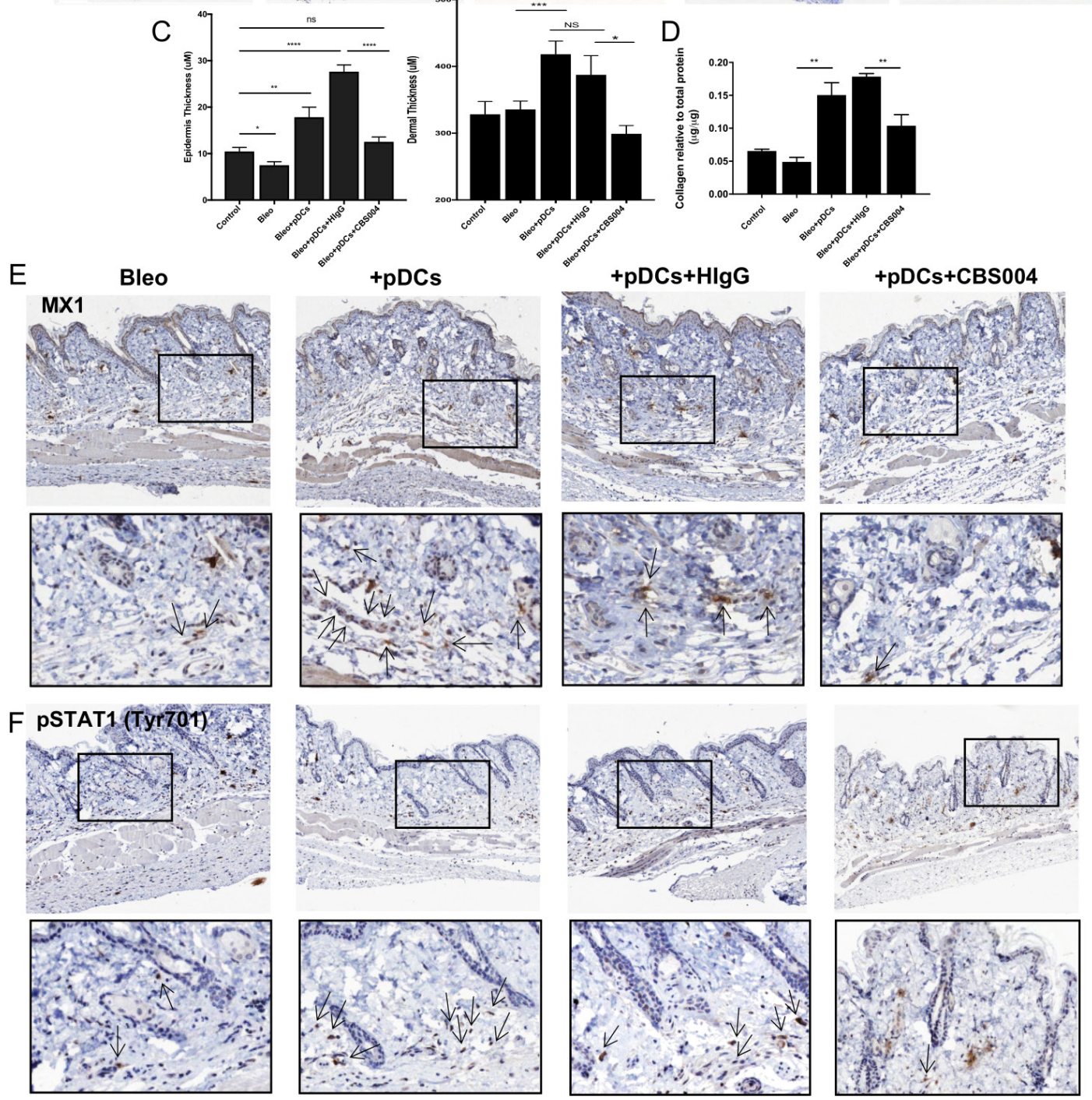

Figure 4 XenoSCID with human pDC increased the pro-fibrotic skin response to bleomycin treatment in a BDCA2-dependent manner. Intravenous tail injection of $2.5 \times 10^{5}$ human purified pDC and intraperitoneal injection of CBS004 mAb (5 mg/kg) or CTR human lgG into NOD SCID mice treated with Bleo or PBS injections (online supplemental figure S5A); systematic diagram and timeline, with five different treatment conditions consisting of PBS/CTR, Bleo, Bleo+pDC, Bleo+pDC+CBS004 and Bleo+pDC+HlgG, each in triplicate. Treated skin was harvested using a $3 \mathrm{~mm}$ punch biopsy and processed for H\&E (A) and MT staining (B). (C) Epidermis and dermal thickness were measured from 20 areas in each condition. (D) An additional punch biopsy was taken and used to extract protein. Total collagen content measured by Sircol ${ }^{\mathrm{TM}}$ assay and shown relative to total protein concentration. Results represented as means \pm SEM of triplicate experiments. Statistical significance was evaluated using paired two-tailed $t$-test. $(E, F)$ IHC analysis of MX1 and PSTAT1 (Tyr701) representative images with zoomed in areas, arrows highlight positively stained cells. ${ }^{*} P<0.05$, ${ }^{*} P<0.01$, ${ }^{* * *} \mathrm{P}<0.001$. Bleo, bleomycin; CTR, control; HIgG, human IgG; IHC, immunohistochemistry; MT, Masson trichrome; NS, no significance; NOD SCID, nonobese diabetic severe combined immunodeficiency; PBS, phosphate buffered saline; pDC, plasmacytoid dendritic cell; SEM, standard error of mean. 
as shown by partially retained fatty layer, as well as no significant differences observed in skin thickness and collagen content compared with control (figure 4A-D). Furthermore, no significant increase in MX1 protein expression was observed (online supplemental figure S5B,C). Injection of human pDC resulted in bleomycin-induced loss of all subdermal fat, along with increased collagen formation and a $40 \%$ increase in overall skin thickness (figure 4A-D). The fibrotic response was associated with type I IFN signalling activation as MX1 and pSTAT1 (Tyr701) protein expression was increased (figure 4E,F and online supplemental figure S5B,C).

To determine the therapeutic implications of BDCA2-targeting, bleomycin-XenoSCID were treated with intraperitoneal injection of CBS004 or HIgG (online supplemental figure S5A). pDC-induced skin fibrosis was dramatically reduced by BDCA2targeting as demonstrated by the retention of some fatty layer tissue, similar to bleomycin-only treated mice, a 2 -fold reduction in dermal and epidermal thicknesses and 1.5-fold reduction in collagen content compared with HIgG (figure 4A-D). Furthermore, reduction in MX1 and specific pSTAT1 dermal fibroblast (based on morphology) protein expression was observed compared with HIgG treatment (figure 4E,F and online supplemental figure S5B,C).

Overall, our xenotransplant models clearly show that human pDC have a crucial and direct role in skin inflammation and fibrosis and highlight $\mathrm{pDC}$ as a viable therapeutic target for SSc.

\section{DISCUSSION}

In the past decade, substantial evidence has pointed to the involvement of $\mathrm{pDC}$ in the pathogenesis of many IMID, including SSc. ${ }^{24-28}$ Nevertheless, research on pDC has focused only on indirect evidence using ex vivo human samples or mouse models. A recent key study supports that mouse $\mathrm{pDC}$ have a pathogenic role in fibrosis, as pDC depletion reduced bleomycin-induced IFN-I stimulated transcripts and prevented fibrosis. ${ }^{24}$ Due to key differences between mouse and human $\mathrm{pDC}$, functions determined in mouse models may not be fully transferable to human pDC. ${ }^{13} 37-39$ In our study, we overcame these challenges by implementing novel preclinical models of human pDC function in vitro and in vivo. The development of our xenograft models has allowed for the first time to study the role of circulating human $\mathrm{pDC}$ within inflammation and fibrosis. Furthermore, by using CBS004, we were able to study the efficacy of specifically attenuating human $\mathrm{pDC}$ function by BDCA2-targeting.

Our study shows that TLR stimulation of $\mathrm{pDC}$ activates a gene expression profile mapping to activation of inflammation, JAK/ STAT, NF-kB and angiogenesis pathways, predicting a greater biological relevance of $\mathrm{pDC}$ activation in IMID. A time course of ODN stimulation would be beneficial in the future to determine the transcriptome over time and to ensure key gene expression has not been overshadowed, as well as determining the effect of restimulation of previously stimulated $\mathrm{pDC} .{ }^{50}$ Crucially, we have shown that BDCA2-targeting is effective at blocking pDC IFN production, as well as the ODN-induced transcriptome. Furthermore, we show that BDCA2-targeting strongly suppresses the differentiation of IFN-secreting CD274 ${ }^{+} \mathrm{pDC}^{17}$ with a prevalent differentiation towards $\mathrm{CD} 274^{-} \mathrm{CD} 80^{+} \mathrm{pDC}$. Further functional studies will shed light on the effects of BDCA2-targeting on T-cell costimulation, which has been suggested to be affected by $\mathrm{CD} 274^{-} \mathrm{CD} 80^{+} \mathrm{pDC}^{17}$

Our preclinical organotypic model of human skin allowed us to show target cell activation by TLR-activated $\mathrm{pDC}$ and further supports the biological relevance of BDCA2 inhibition. Our
XenoSCID model using human pDC greatly expanded this observation. By inducing local TLR-activated skin, we have shown that human $\mathrm{pDC}$ can migrate efficiently into Aldara-treated skin and enhance mouse IFN response, as seen in patients with SSc. ${ }^{24-28}$ Aldara treatment alone increased a small increase in mouse IFN skin response. pDC can be found in mouse blood and lymphoid tissue of NOD SCID mice but are undetectable in skin biopsies either at rest or following imiquimod application for 24 hours, ${ }^{51}$ indicating our observations are unlikely to be caused by host pDC. Furthermore, we clearly see that human pDC are capable of inducing IFN and fibrotic skin response when introduced into our bleomycin mouse model. While these data directly support a pro-fibrotic effect of $\mathrm{pDC}$ in response to bleomycin, they do not directly show that IFN is driving this effect. As shown in our in vitro data, $\mathrm{pDC}$ produce other cytokines that could plausibly drive the tissue fibrosis in this model. Nevertheless, BDAC2targeting of $\mathrm{pDC}$ in situ prevented the pathogenic responses to proinflammatory and pro-fibrotic stimuli, identifying specific pDC targeting to be a viable therapeutic application for SSc. Our data are supported by similar observations seen when mouse pDC were depleted in a bleomycin-induced fibrotic model of SSc and when specific BDCA2-targeting of resident human pDC in a xenograft Psoriasis model prevented progression into psoriatic skin. ${ }^{192452}$

The development of our XenoSCID model is a novel tool that can be used to study the biology of human pDC in mice and can be applied in the research of other IMID affecting the skin, such as psoriasis or SLE. A limitation of this approach is the lack of adaptive immune response in these animals. Therefore, the consequence of $\mathrm{pDC}$ inhibition in a competent immune system remains unknown. Nevertheless, the studies from Rowland et al showed that in a mouse model of SLE, elimination of pDC strongly impaired expansion and activation of $\mathrm{T}$ and $\mathrm{B}$ cells. ${ }^{21}$ In this context, xenotransplant models of human PBMC with and without pDC depletion would be extremely informative although falling beyond the scope of this study.

Together, our data indicate that human pDC, and their cytokine production, are a key cell type in the pathogenesis of SSc. As shown in our in vitro and in vivo models, BDCA2-targeting of human $\mathrm{pDC}$ can reduce ISG response and inflammation, as well as prevent fibrosis. For effective therapeutic application, stratification of patients for those with pDC infiltration and higher IFN score should aid responsiveness to BDCA2 suppression of fibrosis.

\section{MATERIAL AND METHODS}

Detailed description of experimental methods is available in online supplemental file 1 .

\footnotetext{
Author affiliations

${ }^{1}$ Leeds Institute of Rheumatic and Musculoskeletal Medicine, Faculty of Medicine and Health, University of Leeds, Leeds, UK

${ }^{2}$ Scleroderma Programme, NIHR Leeds Musculoskeletal Biomedical Research Centre, Leeds, UK

${ }^{3}$ Leeds Institute of Medical Research, Faculty of Medicine and Health, University of Leeds, Leeds, UK

${ }^{4}$ Rheumatology Department of Lucania, Rheumatology Institute of Lucania (IReL), Potenza, Italy

${ }^{5}$ Department of Internal Medicine III, University of Erlangen, Erlangen, Germany

${ }^{6}$ Capella Biosciences Ltd, Cambridge, UK

${ }^{7}$ Department of Biosciences, Nottingham Trent University, Nottingham, UK

${ }^{8} \mathrm{Clinical}$ Pathology Department, Faculty of Medicine, Mansoura University, Mansoura Egypt

${ }^{9}$ Virus Host Interactions Team, Section of Infection and Immunity, University of Leeds Faculty of Medicine and Health, Leeds, UK
} 
Twitter Clive S McKimmie @VHIT_mckimmie and Francesco Del Galdo @ delgaldoFrances

Acknowledgements Histology was supported by the faculty of medicine and the health histology department, in particular by Filomena Esteves.

Contributors FDG, CSM, YME-S, SH and RLR designed the study. RLR, CC, GM and YME-S performed the experiments and analysed the data, with additional help from CWW, IG and AC. RNAseq analysis was performed by IMC and AA. JHWD gave conceptual advice and helped with the data interpretation and manuscript draft. RLR and FDG wrote the manuscript draft. All authors contributed to the draft review.

Funding Study was funded by research grant to FDG; RLR and FDG are supported by Kennedy Trust Program Foundation Grant, and FDG supported also by the NIHR Biomedical Research Centre. CWW is a Susan Cheney Scleroderma Research Fellow.

Competing interests $\mathrm{SH}$ is an employee of Capella Bioscience, which holds a patent for CBS004 (GB1911188.9)

Patient consent for publication Not required.

Ethics approval Ethical approval for work carried out on patient samples was granted through strike scleroderma to FDG HRA number 15.NE.0211. All animal experiments were undertaken with permission of the local Animal Welfare, Ethics and Research Board and the Home Office (licence PA7CF4E75).

Provenance and peer review Not commissioned; externally peer reviewed.

Data availability statement The authors confirm that the data supporting the findings of this study are available within the article and its supplementary materials.

Supplemental material This content has been supplied by the author(s). It has not been vetted by BMJ Publishing Group Limited (BMJ) and may not have been peer-reviewed. Any opinions or recommendations discussed are solely those of the author(s) and are not endorsed by BMJ. BMJ disclaims all liability and responsibility arising from any reliance placed on the content. Where the content includes any translated material, BMJ does not warrant the accuracy and reliability of the translations (including but not limited to local regulations, clinical guidelines, terminology, drug names and drug dosages), and is not responsible for any error and/or omissions arising from translation and adaptation or otherwise.

Open access This is an open access article distributed in accordance with the Creative Commons Attribution Non Commercial (CC BY-NC 4.0) license, which permits others to distribute, remix, adapt, build upon this work non-commercially, and license their derivative works on different terms, provided the original work is properly cited, appropriate credit is given, any changes made indicated, and the use is non-commercial. See: http://creativecommons.org/licenses/by-nc/4.0/.

\section{ORCID iDs}

Rebecca L Ross http://orcid.org/0000-0002-8528-2283

Antonio Carriero http://orcid.org/0000-0002-3112-6488

Jörg H W Distler http://orcid.org/0000-0001-7408-9333

Yasser M El-Sherbiny http://orcid.org/0000-0003-4791-3475

Francesco Del Galdo http://orcid.org/0000-0002-8528-2283

\section{REFERENCES}

1 Colonna M, Trinchieri G, Liu Y-J. Plasmacytoid dendritic cells in immunity. Nat Immunol 2004;5:1219-26.

2 Gilliet M, Cao W, Liu Y-J. Plasmacytoid dendritic cells: sensing nucleic acids in viral infection and autoimmune diseases. Nat Rev Immunol 2008:8:594-606.

3 Duramad O, Fearon KL, Chan JH, et al. II-10 regulates plasmacytoid dendritic cell response to $\mathrm{CpG}$-containing immunostimulatory sequences. Blood 2003;102:4487-92.

4 Siegal FP, Kadowaki N, Shodell M, et al. The nature of the principal type 1 interferonproducing cells in human blood. Science 1999;284:1835-7.

5 Swiecki M, Colonna M. Unraveling the functions of plasmacytoid dendritic cells during viral infections, autoimmunity, and tolerance. Immunol Rev 2010;234:142-62.

6 Guiducci C, Coffman RL, Barrat FJ. Signalling pathways leading to IFN-alpha production in human plasmacytoid dendritic cell and the possible use of agonists or antagonists of TLR7 and TLR9 in clinical indications. J Intern Med 2009:265:43-57.

7 Reizis B. Plasmacytoid dendritic cells: development, regulation, and function. Immunity 2019;50:37-50.

8 Swiecki M, Colonna M. The multifaceted biology of plasmacytoid dendritic cells. Nat Rev Immunol 2015;15:471-85.

9 Barrat FJ, Elkon KB, Fitzgerald KA. Importance of nucleic acid recognition in inflammation and autoimmunity. Annu Rev Med 2016;67:323-36.

10 Barrat FJ, Meeker T, Gregorio J, et al. Nucleic acids of mammalian origin can act as endogenous ligands for Toll-like receptors and may promote systemic lupus erythematosus. J Exp Med 2005;202:1131-9.

11 Hagberg N, Rönnblom L. Systemic lupus erythematosus--a disease with a dysregulated type I interferon system. Scand I Immunol 2015;82:199-207.

12 Pelka K, Shibata T, Miyake K, et al. Nucleic acid-sensing TLRs and autoimmunity: novel insights from structural and cell biology. Immunol Rev 2016;269:60-75.
13 Barrat FJ, Crow MK, Ivashkiv LB. Interferon target-gene expression and epigenomic signatures in health and disease. Nat Immunol 2019;20:1574-83.

14 Hua J, Kirou K, Lee C, et al. Functional assay of type I interferon in systemic lupus erythematosus plasma and association with anti-RNA binding protein autoantibodies. Arthritis Rheum 2006;54:1906-16

15 Lövgren T, Eloranta M-L, Båve U, et al. Induction of interferon-alpha production in plasmacytoid dendritic cells by immune complexes containing nucleic acid released by necrotic or late apoptotic cells and lupus lgG. Arthritis Rheum 2004;50:1861-72.

16 Lande R, Lee EY, Palazzo R, et al. CXCL4 assembles DNA into liquid crystalline complexes to amplify TLR9-mediated interferon- $\alpha$ production in systemic sclerosis. Nat Commun 2019;10:1731

17 Alculumbre SG, Saint-André V, Di Domizio J, et al. Diversification of human plasmacytoid predendritic cells in response to a single stimulus. Nat Immunol 2018;19:63-75.

18 Yao Y, Richman L, Morehouse C, et al. Type I interferon: potential therapeutic target for psoriasis? PLoS One 2008;3:e2737.

19 Nestle FO, Conrad C, Tun-Kyi A, et al. Plasmacytoid predendritic cells initiate psoriasis through interferon-alpha production. J Exp Med 2005;202:135-43.

20 Obermoser G, Pascual V. The interferon-alpha signature of systemic lupus erythematosus. Lupus 2010;19:1012-9.

21 Rowland SL, Riggs JM, Gilfillan S, et al. Early, transient depletion of plasmacytoid dendritic cells ameliorates autoimmunity in a lupus model. J Exp Med 2014:211:1977-91

22 Sisirak V, Ganguly D, Lewis KL, et al. Genetic evidence for the role of plasmacytoid dendritic cells in systemic lupus erythematosus. J Exp Med 2014;211:1969-76.

23 Varga J, Marangoni RG. Systemic sclerosis in 2016: dermal white adipose tissue implicated in SSC pathogenesis. Nat Rev Rheumatol 2017:13:71-2.

24 Ah Kioon MD, Tripodo C, Fernandez D, et al. Plasmacytoid dendritic cells promote systemic sclerosis with a key role for TLR8. Sci Trans/ Med 2018;10. doi:10.1126/ scitransImed.aam8458. [Epub ahead of print: 10 Jan 2018].

25 van Bon L, Affandi AJ, Broen J, et al. Proteome-Wide analysis and CXCL4 as a biomarker in systemic sclerosis. N Eng/ J Med 2014;370:433-43.

26 Barrat FJ, Lu TT. Role of type I interferons and innate immunity in systemic sclerosis: unbalanced activities on distinct cell types? Curr Opin Rheumatol 2019;31:569-75.

27 Liu X, Mayes MD, Tan FK, et al. Correlation of interferon-inducible chemokine plasma levels with disease severity in systemic sclerosis. Arthritis Rheum 2013;65:226-35.

28 Brkic Z, van Bon L, Cossu M, et al. The interferon type I signature is present in systemic sclerosis before overt fibrosis and might contribute to its pathogenesis through high BAFF gene expression and high collagen synthesis. Ann Rheum Dis 2016:75:1567-73

29 Blasius AL, Colonna M. Sampling and signaling in plasmacytoid dendritic cells: the potential roles of Siglec-H. Trends Immunol 2006;27:255-60.

30 Dzionek A, Sohma Y, Nagafune J, et al. BDCA-2, a novel plasmacytoid dendritic cell-specific type II C-type lectin, mediates antigen capture and is a potent inhibitor of interferon alpha/beta induction. J Exp Med 2001:194:1823-34.

31 Crocker PR, Paulson JC, Varki A. Siglecs and their roles in the immune system. Nat Rev Immunol 2007:7:255-66.

32 Cao W, Zhang L, Rosen DB, et al. BDCA2/Fc epsilon RI gamma complex signals through a novel BCR-like pathway in human plasmacytoid dendritic cells. PLoS Biol 2007:5:e248.

33 Fanning SL, George TC, Feng D, et al. Receptor cross-linking on human plasmacytoid dendritic cells leads to the regulation of IFN-alpha production. J Immunol 2006; 177:5829-39.

34 Röck J, Schneider E, Grün JR, et al. CD303 (BDCA-2) signals in plasmacytoid dendritic cells via a BCR-like signalosome involving Syk, Slp65 and PLCgamma2. Eur J Immunol 2007:37:3564-75.

35 Furie R, Werth VP, Merola JF, et al. Monoclonal antibody targeting BDCA2 ameliorates skin lesions in systemic lupus erythematosus. J Clin Invest 2019:129:1359-71.

36 Pellerin A, Otero K, Czerkowicz JM, et al. Anti-BDCA2 monoclonal antibody inhibits plasmacytoid dendritic cell activation through $\mathrm{Fc}$-dependent and $\mathrm{Fc}$-independent mechanisms. EMBO Mol Med 2015;7:464-76.

37 Hartwig T, Zwicky P, Schreiner B, et al. Regulatory T cells restrain pathogenic T helper cells during skin inflammation. Cell Rep 2018;25:3564-72.

38 Walter A, Schäfer M, Cecconi V, et al. Aldara activates TLR7-independent immune defence. Nat Commun 2013:4:1560.

39 Friedberg JW, Kim H, McCauley M, et al. Combination immunotherapy with a CpG oligonucleotide (1018 ISS) and rituximab in patients with non-Hodgkin lymphoma: increased interferon-alpha/beta-inducible gene expression, without significant toxicity. Blood 2005:105:489-95.

40 Johnson ME, Mahoney JM, Taroni J, et al. Experimentally-derived fibroblast gene signatures identify molecular pathways associated with distinct subsets of systemic sclerosis patients in three independent cohorts. PLoS One 2015;10:e0114017.

41 Assassi S, Swindell WR, Wu M, et al. Dissecting the heterogeneity of skin gene expression patterns in systemic sclerosis. Arthritis Rheumatol 2015;67:3016-26.

42 Rice LM, Ziemek J, Stratton EA, et al. A longitudinal biomarker for the extent of skin disease in patients with diffuse cutaneous systemic sclerosis. Arthritis Rheumatol 2015;67:3004-15 
43 Jego G, Palucka AK, Blanck J-P, et al. Plasmacytoid dendritic cells induce plasma cell differentiation through type I interferon and interleukin 6. Immunity 2003;19:225-34.

44 Toyama-Sorimachi N, Kitamura F, Habuchi H, et al. Widespread expression of chondroitin sulfate-type serglycins with CD44 binding ability in hematopoietic cells. J Biol Chem 1997:272:26714-9.

45 Toyama-Sorimachi N, Sorimachi H, Tobita Y, et al. A novel ligand for CD44 is serglycin a hematopoietic cell lineage-specific proteoglycan. Possible involvement in lymphoid cell adherence and activation. J Biol Chem 1995;270:7437-44.

46 Guo J-Y, Hsu H-S, Tyan S-W, et al. Serglycin in tumor microenvironment promotes non-small cell lung cancer aggressiveness in a CD44-dependent manner. Oncogene 2017;36:2457-71.

47 Korpetinou A, Skandalis SS, Labropoulou VT, et al. Serglycin: at the crossroad of inflammation and malignancy. Front Oncol 2014;3:327.
48 Ploeger DT, Hosper NA, Schipper M, et al. Cell plasticity in wound healing: paracrine factors of M1/ M2 polarized macrophages influence the phenotypical state of dermal fibroblasts. Cell Commun Signal 2013;11:29.

49 Liu Y-J. IPC: professional type 1 interferon-producing cells and plasmacytoid dendritic cell precursors. Annu Rev Immunol 2005;23:275-306

50 Ito T, Kanzler H, Duramad O, et al. Specialization, kinetics, and repertoire of type 1 interferon responses by human plasmacytoid predendritic cells. Blood 2006:107:2423-31.

51 Bryden SR, Pingen M, Lefteri DA, et al. Pan-viral protection against arboviruses by activating skin macrophages at the inoculation site. Sci Trans/ Med 2020;12. doi:10.1126/scitranslmed.aax2421. [Epub ahead of print: 22 Jan 2020].

52 Boyman 0, Hefti HP, Conrad C, et al. Spontaneous development of psoriasis in a new animal model shows an essential role for resident T cells and tumor necrosis factoralpha. J Exp Med 2004;199:731-6. 